\title{
A counterrotating central component in the barred galaxy NGC 5728
}

\author{
F. Prada ${ }^{1}$ and C. M. Gutiérrez ${ }^{2}$ \\ ${ }^{1}$ Instituto de Astronomía, UNAM, 22830 Ensenada, Mexico. \\ ${ }^{2}$ Instituto de Astrofísica de Canarias, 38200 La Laguna, Tenerife, Spain.
}

Received ——; accepted — 


\begin{abstract}
We present a detailed study of the stellar kinematics in the barred galaxy NGC 5728 based on I-band photometry and long-slit spectroscopic observations in the region of the near-IR Ca II triplet. The analysis of the stellar line-of-sight velocity distribution (LOSVD) has revealed, in the central regions of the bar, the presence of a cold (v/ $\sigma \sim 2.5)$ prograde $S$-shaped velocity component that coexists in the central $4 \mathrm{kpc}$ with a fainter and hotter $(\mathrm{v} / \sigma \sim 0.5)$ counterrotating component. Beyond $4 \mathrm{kpc}$ from the nucleus the LOSVD shows the stellar bar kinematics. The comparison of the radial surface brightness profile of the velocity components with that obtained from an I-band image shows that the counterrotating core follows a $\mathrm{r}^{1 / 4}$ profile, while the $S$-shaped component does not follows the flat bar surface brightness profile. Several possible scenarios accounting for such kinematic signatures found in the center of the bar in NGC 5728 are discussed.

The data presented in this paper shows for the first time the presence of extended retrograde motions in barred systems which, together with previous discoveries seems to indicate that the stellar counterrotation is a phenomenon present all along the Hubble sequence.
\end{abstract}

Subject headings: galaxies: individual (NGC 5728) — galaxies: kinematics and dynamics galaxies: structure - galaxies: formation 


\section{INTRODUCTION}

It has been reported in the last few years the discovery of counterrotating cores in elliptical galaxies (Franx \& Illingworth 1988; Bender et al. 1994; Rix \& White 1992) and the presence of extended retrograde motions in the discs of early type spirals (Rix et al. 1992; Merrifield \& Kuijken 1994; Bertola et al. 1996). This phenomenon is also present in the Sb galaxy NGC 7331, where the bulge rotates opposite to the disc (Prada et al. 1996). The origin of the stellar counterrotation has been extensively studied and generally attributed to the accretion of a small satellite galaxy or gradual infall of gas in a retrograde orbit (e.g. Merrifield \& Kuijken 1994; Thakar \& Ryden 1996). However, other alternatives are possible in terms of dynamical instabilities (e.g. Wozniak \& Pfenniger 1997), that make it still unclear whether the causes of counterrotation are of external or internal origin. Up to now no observational evidence exists of extended stellar counterrotation in barred galaxies, whose discovery would represent a challenge to understand the significance of counterrotation throughout the Hubble sequence. Having this in mind, we decided to conduct a photometric and spectroscospic study of the SAB(r)a galaxy NGC 5728 (de Vaucouleurs et al. 1991). As a result of this analysis it will be shown here that extended retrograde motions can also be present in

barred systems. In combination with previous observations, our results demonstrate that counterrotation is present all along the Hubble sequence; the study of this phenomenon could thus sheds light to understand the mechanisms and the evolution of galaxy formation.

The paper is organized as follows: Section 2 gives details of the observations and data reduction. Section 3 presents a brief description of the structure of the galaxy. Section 4 describes the analysis of the stellar line-of-sight velocity distribution (LOSVD). Section 5 discuss on the nature of the kinematical components present in this galaxy, and finally Section 6 gives the discussions and conclusions of this work.

\section{OBSERVATIONS AND DATA REDUCTION}

Long-slit spectroscopic observations of NGC 5728 were performed on several runs with the double arm ISIS spectrograph on the 4.2m WHT at La Palma. In July 1996, the slit, 1.3" wide, was placed along p.a. $30^{\circ}$, close to the bar major-axis (p.a. $33^{\circ}$, Schommer et al. 1988). We used the red arm of ISIS with a 1200 $l / \mathrm{mm}$ grating centered at $8750 \AA$ including the near IR CaII triplet. The dispersion was $0.38 \AA \cdot$ pixel $^{-1}$. Two consecutive $1800 \mathrm{~s}$ exposures were recorded. As velocity template we observed the KIII HR 5826 star. The seeing was $1.4^{\prime \prime}$. A similar setup was used in a previous run (February 1995), this time a $1^{\prime \prime}$ slit wide was placed along p.a. $86^{\circ}$, and $23^{\circ}$. A $600 \mathrm{l} / \mathrm{mm}$ grating was used giving a dispersion of $0.79 \AA$ Apixel ${ }^{-1}$. 
Two $1800 \mathrm{~s}$ frames were taken centered at $8750 \AA$ for p.a. $86^{\circ}$, and one $1800 \mathrm{~s}$ exposure for p.a. $23^{\circ}$. The G8 giant HR 7753 star was observed as velocity template in this run. The seeing was $0.9^{\prime \prime}$. In both runs the slit length was $4^{\prime}$ and centered on the apparent optical nucleus of NGC 5728. The detector was a $1024 \times 1024$ TEK CCD giving $0.36^{\prime \prime}$ pixel $^{-1}$. The debias, flatfielding, wavelength calibration and sky subtraction was done in the standard way using the IRAF package.

An I-band CCD image of NGC 5728 was taken with the BroCam CCD camera on the 2.5m NOT at La Palma for the analysis of the morphology and comparison with the stellar kinematics. These observations were done in August 1996 with a seeing of $0.7^{\prime \prime}$. The camera uses a $1024 \times 1024$ TEK CCD with 0.176 ${ }^{\prime \prime}$. pixel $^{-1}$. In total, we recorded a 40 minutes exposure. Figure 1 (Plate 1) presents this image; the bar major-axis and the nuclear features inside the spheroidal component of the galaxy are indicated. Hereafter, we will refer to p.a. $30^{\circ}$ as the bar major-axis.

\section{THE MORPHOLOGY OF NGC 5728}

An isophotal analysis of the I-band image of NGC 5728 was done using Galphot (Jörgensen, Franx, \& Kjaergaard 1992) to determine the surface brightness, ellipticity, position angle and the C4 Fourier coefficient along the semi-major axis radius (see Figure 2). The structure of the galaxy can be summarized as follows: a nuclear feature that appears elongated along p.a. $86^{\circ}$ with a semi-major axis radius of $3.5^{\prime \prime}$ and a maximum ellipticity of 0.5 ; the stellar bar with a p.a. $33^{\circ}$, a length of $\sim 60^{\prime \prime}$, maximum ellipticity of 0.7. Beyond the end of the bar, the ellipticity drops to 0.55 and the disk dominates. A star forming ring is located at $\sim 4^{\prime \prime}$ (see Schommer et al. 1988; Wilson et al. 1993). Interior to this ring the HST images by Wilson et al. (1993) reveal the nuclear feature as the presence of bar-like continuum knots (called ABCD, named from East to West, beeing A and B very blue). This optical continuum light within the ring can be interpreted in terms of a nuclear stellar bar (Shaw et al. 1993; Wozniak et al. 1995), as star formation triggered by the radio ejecta or as scattered light from the active nucleus (see Wilson et al. 1993 for a discussion). The nature of this nuclear feature will be discussed in the following sections.

In the central $\sim 20^{\prime \prime}$, we have a spheroidal component misaligned with respect to the bar major-axis (see Figure 1). This is also shown in the ellipticity profile at $\sim 10^{\prime \prime}$ which has a plateau of $\epsilon \sim 0.3$ and an isophotal twist of $\sim 8^{\circ}$. 


\section{THE STELLAR LINE-OF-SIGHT VELOCITY DISTRIBUTION IN NGC 5728}

The near-IR CaII triplet absorption features were used to determine the stellar LOSVD. The analysis was performed by means of the two-dimensional unresolved Gaussian decomposition algorithm described in Prada et al. (1996), and Prada, Gutiérrez, \& McKeith (1998). The resolution used for the observations presented here, is 2 pixels and 1.4 pixels in the spectral and spatial axes respectively for p.a. $23^{\circ}$ and p.a. $86^{\circ}$, and 3 pixels and 2.2 pixels respectively for the bar major-axis spectra. Figure 3 shows on the left the spectra in several positions along the bar major-axis in the spectral region analyzed, i.e. 8450-8700 $\AA$, together with the modeled spectra, obtained by convolution of the derived LOSVD with the spectrum of the velocity template. The corresponding LOSVD are also plotted on the righthand side of Figure 3. 1-sigma error-bars for each LOSVD were obtained by Monte Carlo simulations in the following way: first, we consider that the difference between the real and the modeled spectra gives an estimation of the error-bar in each point of the real spectra. Then, we simulate spectra considering that the astronomical signal corresponds to the modeled spectra and the noise follows a Gaussian distribution with the errorbar mentioned above. We run again our algorithm for each simulation obtaining the distribution of the LOSVD and taking the dispersion of this distribution as an estimation of the uncertainty in each velocity bin of the LOSVD. Finally, we average over all velocity bins at each position. These error-bars constitute the statistical uncertainty relative to the fit between the original and the recovered spectra and don't take into account any possible effect due for example to the selection of the stellar templates or modeling the shape of the galaxy's continuum.

We would like to mention that CaII in emission is seen in some Seyfert 1 galaxies phisically associated with the BLR, however, there is no strong indication, generally, that the $\mathrm{Ca}^{+}$zone is within a separate dynamical entity such as a disk (see Persson 1988). He also find that the physical conditions are so extreme

in the $\mathrm{Ca}^{+}$zone that the calcium emission cannot be a common feature of the spectra of Seyfert 1 galaxies and it it would be quite rare to see it in a Seyfert 2. NGC 5728 is classified as Seyfert 2 by Philips et al. (1983), therefore we consider that the CaII in emission does not affect our results at all.

The LOSVD in the central $20^{\prime \prime}$ along the bar major-axis shows two distinct components with their velocity means at opposite sides of the systematic velocity, except in the central $2^{\prime \prime}$ where the two components are not present. Beyond a radius of $\sim 12^{\prime \prime}$ at either side of the nucleus the LOSVD consists of a single component. The counterrotation is also confirmed along p.a. $23^{\circ}$ where the better seeing during this run allowed to see the two components in the LOSVD in the central $2^{\prime \prime}$ (see Figure 4). While the LOSVD 
at p.a. $23^{\circ}$ and the bar major axis are very similar in the central positions, there are some differences at distances $\sim 5-7$ arcsecs from the center. Although the position angles are close we believe that at least partially this could be a consequence of the kinematics of the elongated physical structures associated with the barred component.

We have parameterized the LOSVD profiles by fitting one, and where possible two Gaussians; this is enough to describe the major kinematical features. Figure 5 shows the results of this analysis plotting the radial velocities as a function of the radius along the bar major-axis, p.a. $23^{\circ}$ and p.a. $86^{\circ}$. These can be described as a $S$-shaped velocity profile in the central $20^{\prime \prime}$ along the bar major-axis with $\sigma \sim 70 \pm 10 \mathrm{~km}$ $\mathrm{s}^{-1}$ and a maximum velocity of $\sim 170 \mathrm{~km} \mathrm{~s}^{-1}$ at $\pm 5^{\prime \prime}$. Superimposed on this cold component is a fainter counterrotating component that extends from $2^{\prime \prime}$ out to $11^{\prime \prime}$ at either side of the nucleus along the bar major-axis with a $\sigma=95 \pm 10 \mathrm{~km} \mathrm{~s}^{-1}$, and a slow rotation reaching $\sim 90 \mathrm{~km} \mathrm{~s}^{-1}$ at $\pm 5^{\prime \prime}$. In the central $2^{\prime \prime}$, a single Gaussian was fitted to the LOSVD with $\sigma=165 \pm 15 \mathrm{~km} \mathrm{~s}^{-1}$. A similar velocity pattern is also present along p.a. $23^{\circ}$. At this position angle we fit two Gaussians to the LOSVD in central $2^{\prime \prime}$ (see Figure 5 ). Beyond $\sim 20^{\prime \prime}$ radius the velocity linearly rises with radius and the velocity dispersion is $55 \pm 10 \mathrm{~km} \mathrm{~s}^{-1}$. The kinematics in this region can be attributed to the stellar bar (see next section).

The velocities along p.a. $86^{\circ}$ basically describe the overall kinematics seen along the bar majoraxis, only in the central $4^{\prime \prime}$ the LOSVD appears asymmetric. This might suggest the presence of the counterrotating component seen at this position angle, since the fit of a single Gaussian of $\sigma \sim 160 \pm 15 \mathrm{~km}$ $\mathrm{s}^{-1}$ to the LOSVD agrees with the velocity dispersion of a single Gaussian fit to the LOSVD in the very center along the bar major-axis. However, higher spectral resolution data is needed to confirm the presence of the two components along p.a. $86^{\circ}$.

In summary, we have found in the central parts of the $11 \mathrm{kpc}$ (we adopt a scale of $1^{\prime \prime} \sim 190 \mathrm{pc}$ for $\left.\mathrm{H}_{\mathrm{o}}=75 \mathrm{~km} \mathrm{~s}^{-1} \mathrm{Mpc}^{-1}\right)$ bar of NGC 5728 a cold $(\mathrm{v} / \sigma \sim 2.5)$ prograde $S$-shaped velocity component which coexist in the central $4 \mathrm{kpc}$ with a dynamically hot $(\mathrm{v} / \sigma \sim 0.5)$ counterrotating component.

\section{THE NATURE OF THE CENTRAL BAR KINEMATICS}

A comparison between the photometric and the kinematic components found in the center of the bar in NGC 5728, should shed some light onto the nature and dynamics of such structures. For this purpose, we have compared the surface brightness profile for a cut along p.a. $30^{\circ}$ obtained from the I-band image 
with the relative fluxes of the velocity components found in our spectroscopic analysis along this position (see Figure 6).

On the basis of its kinematical and morphological properties we propose to fit the surface brightness profiles of the three velocity components inside $70^{\prime \prime}$ with three components. First, we model the bar by a flat bar profile $\left(I=I_{o} /(1+\exp ((r-b) / a))\right.$, which describes the bar major-axis surface brightness profiles of early type barred galaxies (Elmegreen et al. 1996). The parameters found for NGC 5728 yield a characteristic scale-length of $b=53^{\prime \prime}, a=4.1^{\prime \prime}$ for the region between $30^{\prime \prime}$ and $60^{\prime \prime}$. For the counterrotating component we propose an $r^{1 / 4}$ law for the fit due to the fact that this is a hot slowly rotating component and can be associated with the spheroidal component present in the central $20^{\prime \prime}$ of the galaxy (see section 3). We get a very good fit with an effective radius of $21.8^{\prime \prime}$ for the region between $1^{\prime \prime}$ and $9^{\prime \prime}$. Finally, the brighter component in this region, i.e. the $S$-shaped velocity profile has been fitted by an exponential profile of $4^{\prime \prime}$ scale-length. The sum of the three modeled surface brightness profiles agrees very well with the overall radial surface brightness I-band image cut along the bar major-axis (see Figure 6).

The question now is whether the counterrotating core and/or the $S$-shaped velocity profile represent the kinematics of the bar in the central regions. The $S$-shaped velocity component has the same v/ $\sigma$ as the bar kinematics outside a radius of $20^{\prime \prime}$, but its surface brightness profile does not follow the flat bar surface brightness profile. The reason for this might be the contamination by the nuclear feature and the star-forming ring inside the $5^{\prime \prime}$ radius. Following the discussion by Wilson et al. (1993), we agree with them that the nuclear feature correspond to a blue component that dominates the light in the very central regions (see Figure 5), but does not contribute to the gravitational potential as indicated by its kinematics (see Section 4). The reason why we state that the $S$-shaped component is associated to the barred kinematics is that the gaseous velocity field in NGC 5728 (see Rubin 1980; Schommer et al. 1988) shows also an $S$-shaped feature and follows the stellar kinematics at radii larger than $20^{\prime \prime}$. The gaseous kinematics has been interpreted by Schommer et al. (1988) in terms of models of gas motions in non-axisymmetric potentials and they claim that the star-forming ring and the $S$-shaped velocity field may be related to an inner Lindblad resonance. The gaseous kinematics also shows an expansion of $\sim 400 \mathrm{~km} \mathrm{~s}^{-1}$ in the central $3.5^{\prime \prime}$ but it does not show any counterrotating signature.

However, the $S$-shaped velocity profile can also be interpreted as a very fast rotation due to a heavy mass concentration in the center of the bar in NGC 5728. From the measured velocities in the very center, we can estimate an mass of $\sim 410^{9} \mathrm{M}_{\odot}$ inside a radius of 300 pc for an inclination of $48^{\circ}$ (Schommer et al. 
1988). This mass could be related to its Seyfert nucleus.

\section{DISCUSSION AND CONCLUSIONS}

Several scenarios have been proposed to explain kinematical structures similar to the ones found in NGC 5728: dynamical instabilities, the accretion of a satellite or gas infall with retrograde orbits. In the first case a non-axisymmetric structure such as a bar modifies the stellar orbits, introducing retrograde motions in its center, as shown in numerical simulations by Wozniak \& Pfenniger (1997). In their models, the presence of retrograde orbits (almost circular and perpendicular to the major-axis of the bar) inside the corotation radius, can explain local counterrotation or even could support a counterrotating core. One can expect that, if the mass on such orbits increases, a critical mass ratio can be reached beyond which the dynamics of the counterrotating component are decoupled from the direct bar. In this scenario the presence of the counterrotating core in NGC 5728 could be interpreted as a secondary retrograde bar, and its presence could thus be intrinsic to the primary bar. Indeed, n-body simulations by Friedli (1996) have shown that a counterrotating secondary stellar bar can be stable.

However, we cannot rule out an external origin for the presence of retrograde motions in the center of NGC 5728 as shown by the models of Thakar \& Ryden (1996). They explain the counterrotation of the disks of spirals by the accretion of small satellites or gas with negative angular momentum.

We conclude that the data presented in this paper reveal for the first time the presence of extended retrograde motions in barred galaxies which, together with previous discoveries in ellipticals, early-type spirals and Sb galaxies (see Bertola \& Corsini 1997 for a review), seems to indicate that counterrotation is a phenomenon present all along the Hubble sequence. The scenarios mentioned above could explain these, or alternatively a more general physical mechanism that allows for galaxy transformation processes along the Hubble sequence might be needed. In any case, the relevance of the phenomenon is clear and requires for a deeper understanding, more statistics and two-dimensional spectroscopy.

This research is based on observations obtained at the WHT, operated by the ING and the NOT at the Observatorio del Roque de los Muchachos (La Palma, Spain) of the IAC. We want to thank A. Manchado for taking the NOT image and E. Pérez, H. Zhao and the referee for useful comments on the manuscript. 


\section{REFERENCES}

Bender, R., Saglia, R. P. \& Gerhard, O. E. 1994, MNRAS 269, 785

Bertola, F., Cinzano, P., Corsini, E. M., Pizzella, A., Persic, M. \& Salucci, P. 1996, ApJ, 458, L67

Bertola, F., \& Corsini, E.M. 1997, in Galaxy Interactions at Low and High Redshift, IAU Symposium 186, Kyoto, in press

de Vaucouleurs, G., de Vaucouleurs, A., Corwin, H. G.,jr, Buta, R. J., Paturel, G. \& Fouqué, P. 1991, Third Reference Catalog ue of Bright Galaxies, Springer Verlag

Elmegreen, B., Elmegreen, D. M., Chromey, F. R., Hasselbacher, D. A., \& Bissell, B. A. 1996, AnJ, 111, 2233

Franx, M. \& Illingworth, G. 1988, ApJ, 327, L55

Friedli, D. 1996, A\&A, 312, 761

Jørgensen, I., Franx, M. \& Kjaergaard, P. 1992, A\&A S, 95, 489

Merrifield, M. R. \& Kuijken, K. 1994, ApJ, 432, 575

Persson, S. E. 1988, ApJ, 330, 751

Phillips, M. M., Charles, P. A., \& Baldwin, J. A. 1983, ApJ, 266, 485

Prada, F., Gutiérrez, C. M., Peletier, R. F. \& McKeith, C. D. 1996, ApJ, 463, L9

Prada, F., Gutiérrez, C. M. \& McKeith, C. D. 1998 ApJ, 495, 765

Rix, H.-W. \& White, S. D. M. 1992, MNRAS, 254, 389

Rix, H-W. Franx, M. Fisher, D. \& Illingworth, G. 1992, ApJ, 400, L5

Rubin, V. 1980, ApJ, 238, 808

Shaw, M., Combes, F., Axon, D. J. \& Wright, G. S. 1993, A\&A, 273, 31

Schommer, R. A., Caldwell, N., Wilson, A. S., Baldwin, J. A., Phillips, M. M., Williams, T. B. \& Turtle, A. J. 1988, ApJ, 324, 154

Thakar, A. R. \& Ryden, B. S. 1996, ApJ, 461, 55

Wilson, A. S., Braatz, J. A., Heckman, T. M., Krolik, J. H., \& Miley, G. K. 1993, ApJ, 419, L61

Wozniak, H., Friedli, D., Martinet, L., Martin, P. \& Bratschi, P. 1995, A\&A, 111, 115 
Wozniak, H. \& Pfenniger, D. 1997, A\&A,317,14 


\section{FIGURE CAPTIONS}

Fig. 1.- An I-band grey-scale image of NGC 5728. The NE-SW and E-W arrows indicate the bar major-axis and the nuclear features at p.a. $86^{\circ}$, respectively. Notice the misalignment of the spheroidal component with respect to the stellar bar.

Fig. 2.- Isophotal analysis of the I-band surface photometry of NGC 5728. From top to bottom: the major axis surface brightness profile, the ellipticity profile, the position angle profile, and the C4 Fourier coefficient profile, plotted against the square root of the radius in arcsec.

Fig. 3.- (Left:) The original and modeled spectra in the region of the near-IR CaII triplet at different positions along the bar major-axis in NGC 5728. (Right:) The corresponding LOSVD. 1-sigma error-bars for each LOSVD are shown (see text). SW is at top.

Fig. 4.- A comparison of the LOSVD along the bar major-axis and p.a. $23^{\circ}$. The LOSVD along p.a. $86^{\circ}$ are also shown; West is at top (see text). Error-bars as in Fig. 3.

Fig. 5.- Radial velocities determined by fitting two Gaussians to the LOSVD along the bar major-axis (top), p.a. $23^{\circ}$ (middle), and p.a. $86^{\circ}$ (bottom). Open and filled squares refer to the fainter and brighter components present in the LOSVD. The triangles refer to a single component in the LOSVD. The open triangles in p.a. $86^{\circ}$ refer to the fit of a single Gaussian to the asymmetric LOSVD in the central $3.5^{\prime \prime}$. The dashed line correspond to the continuum at $\sim 8500 \AA$ obtained from the spectroscopic data along p.a. $86^{\circ}$. The letters ABD indicate the position of the features named by Wilson et al. (1993) in the HST continuum images of NGC 5728 .

Fig. 6.- The surface brightness profile of the three velocity components obtained from the LOSVD along the bar major-axis, i.e. the counterrotating core (open squares), the cold $S$-shaped component (filled squares), and the bar (triangles). The open circles represent the sum of the counterrotating and $S$-shaped surface brightness profiles. Also plotted is the surface brightness cut along the bar major-axis obtained from the I-band image (solid line). The dotted lines are the modeled surface brightness profiles for the three velocity components. The dashed line is the sum of these three model components. (see text). 


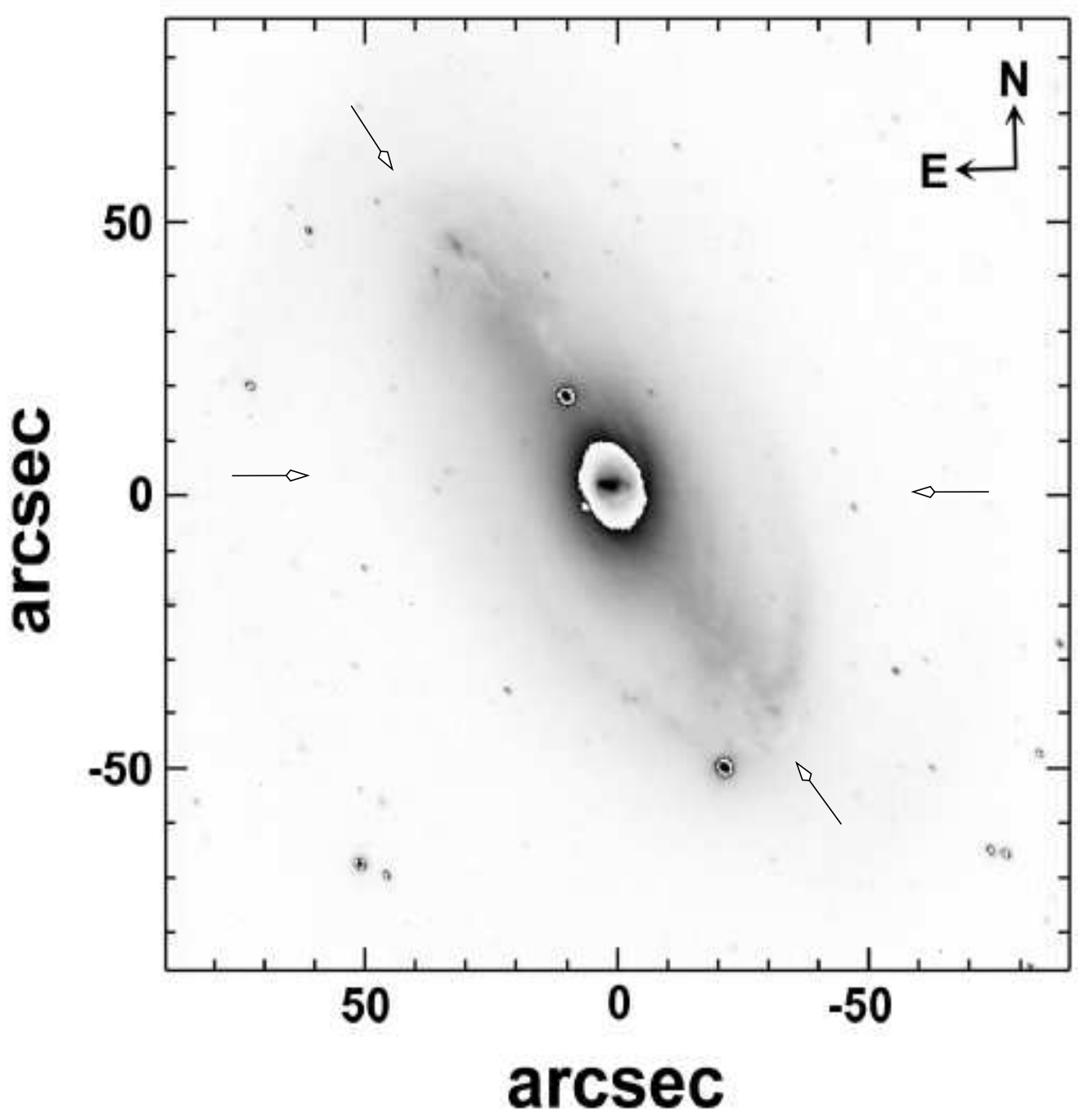




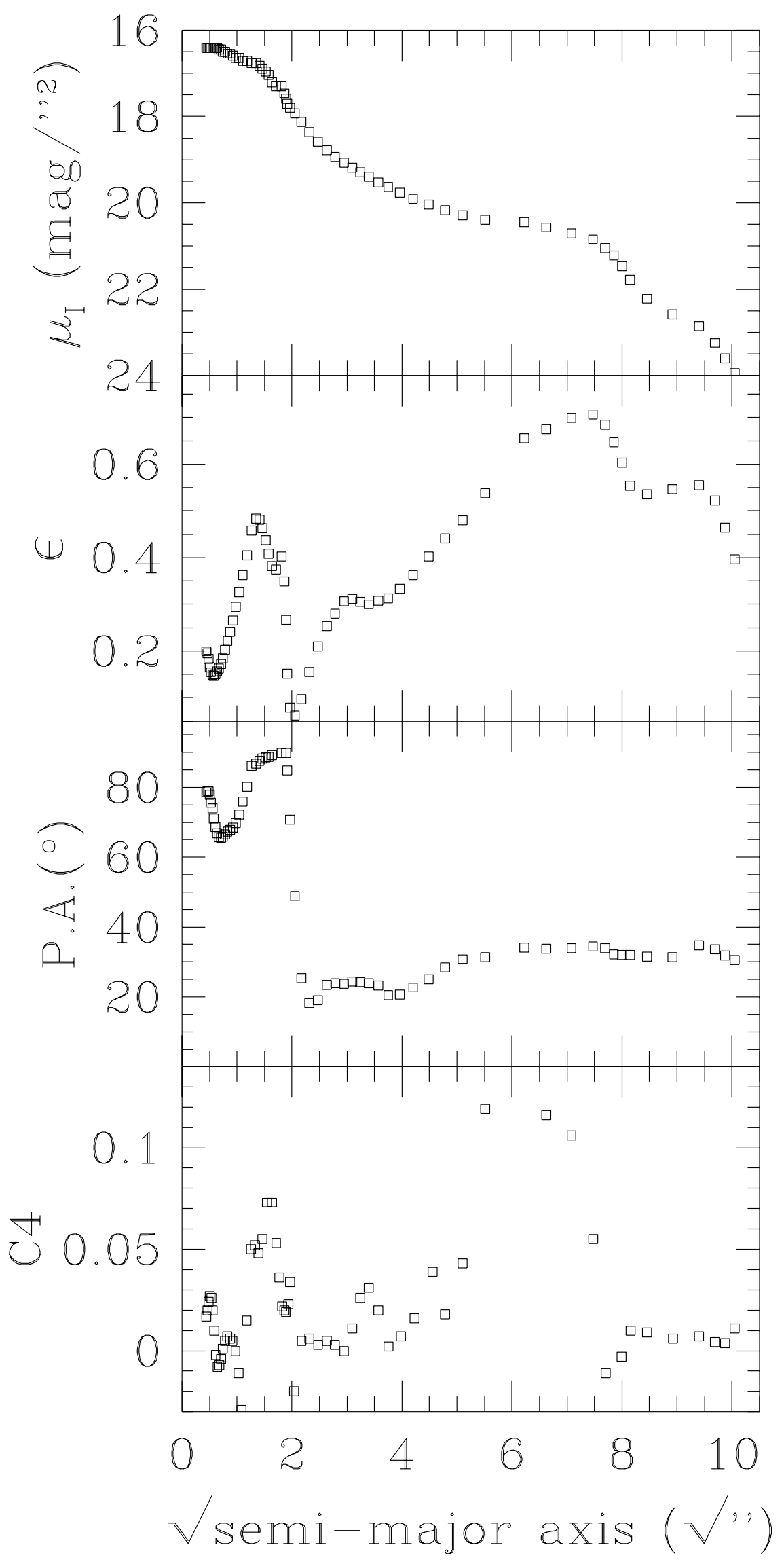




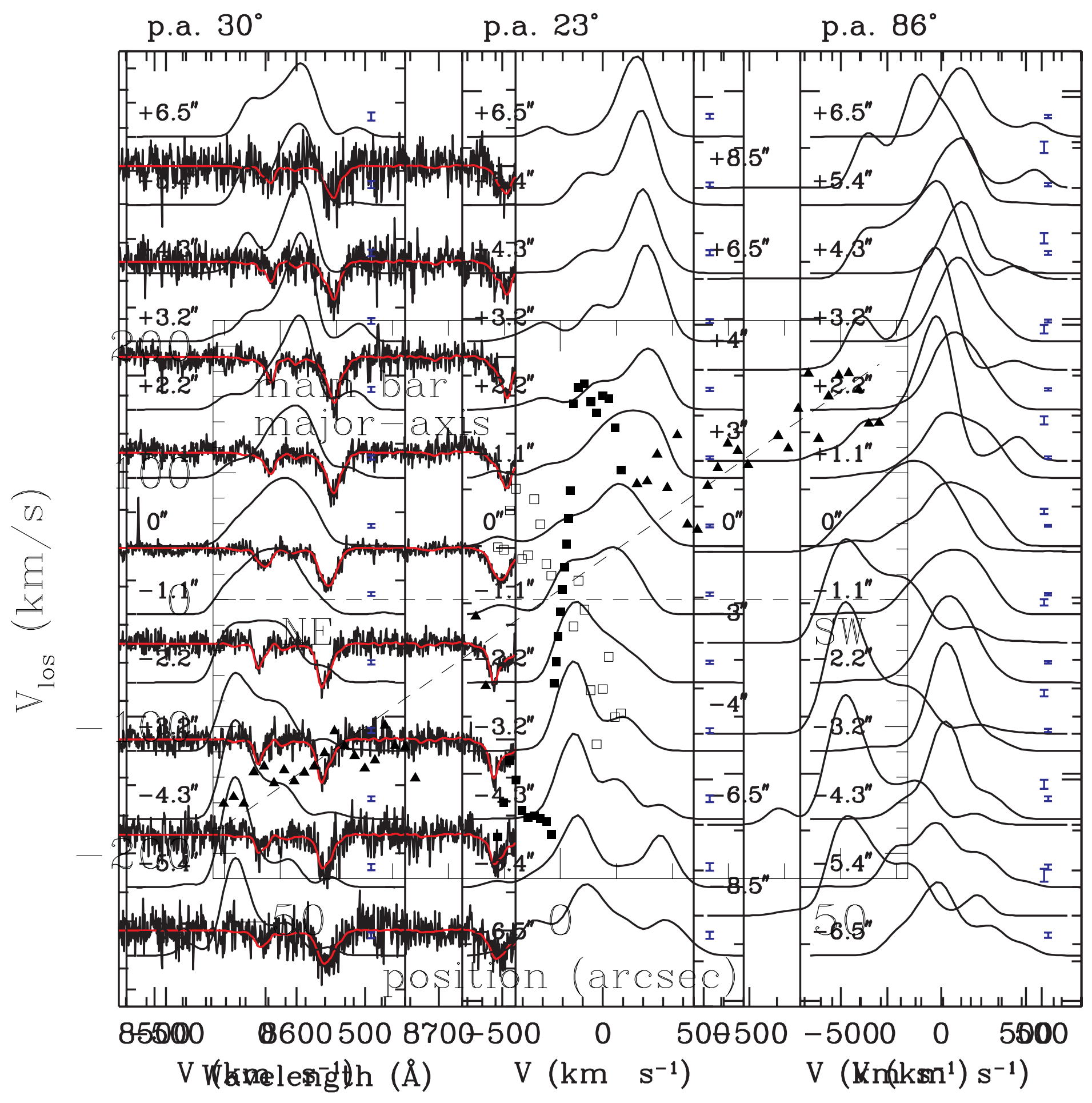




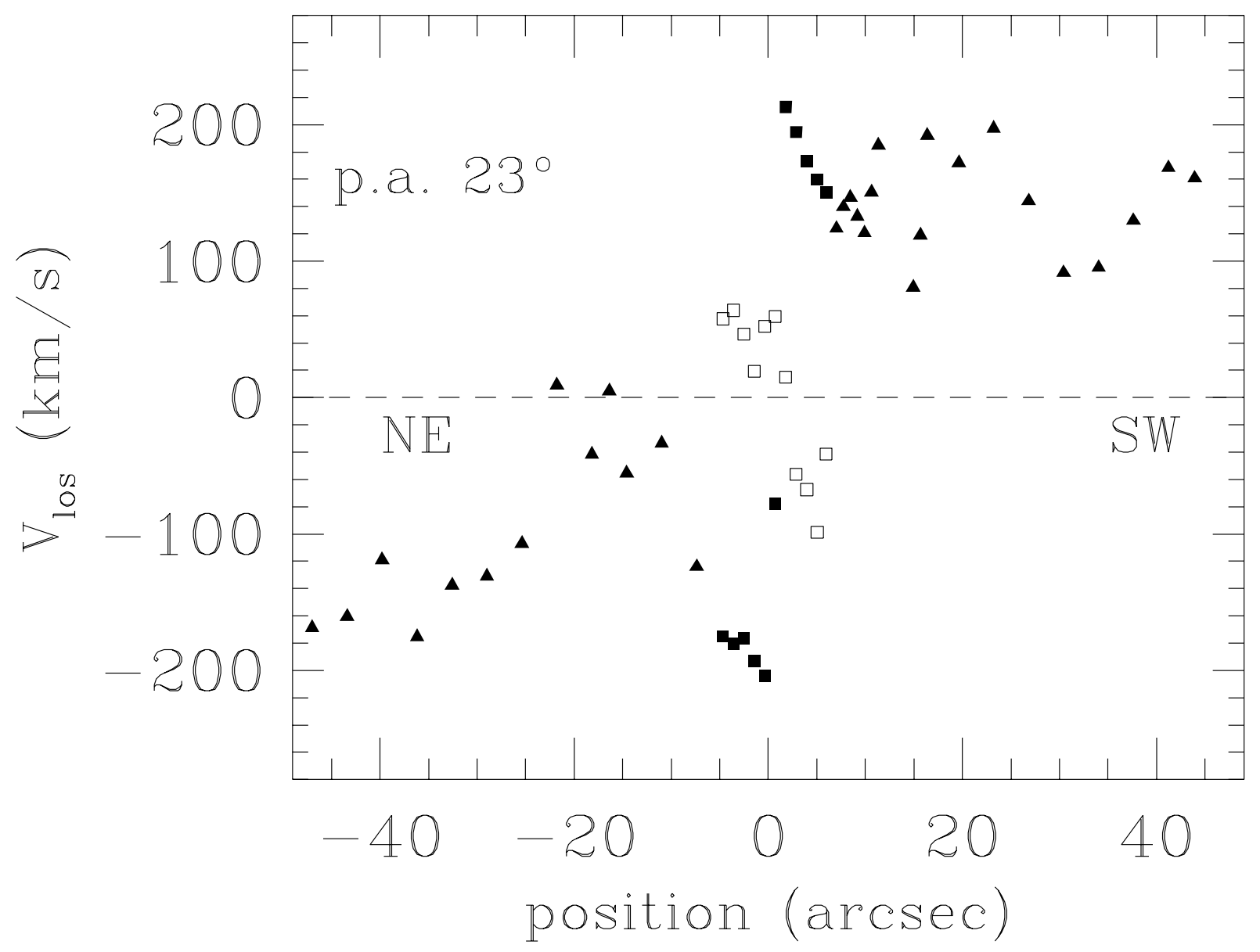




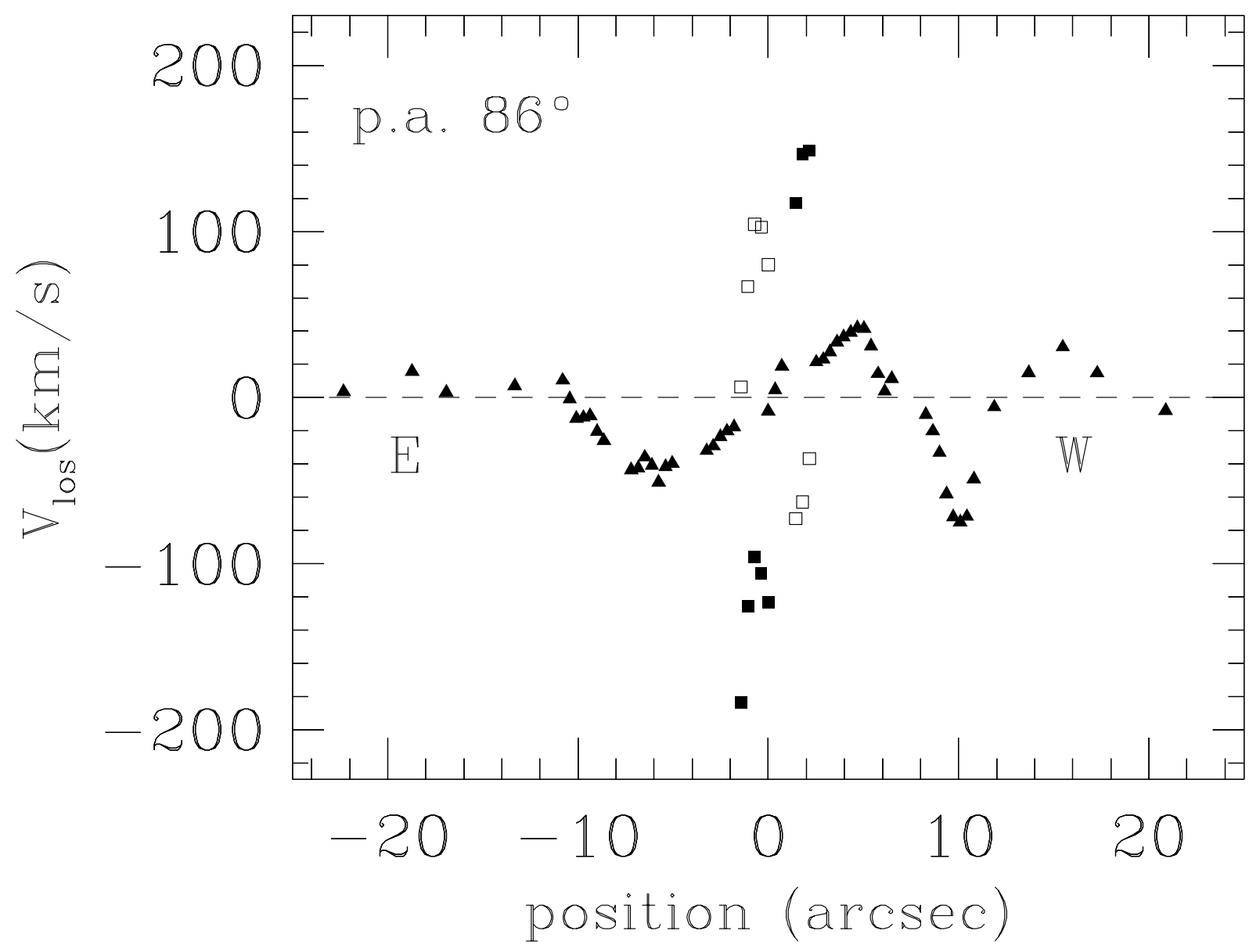




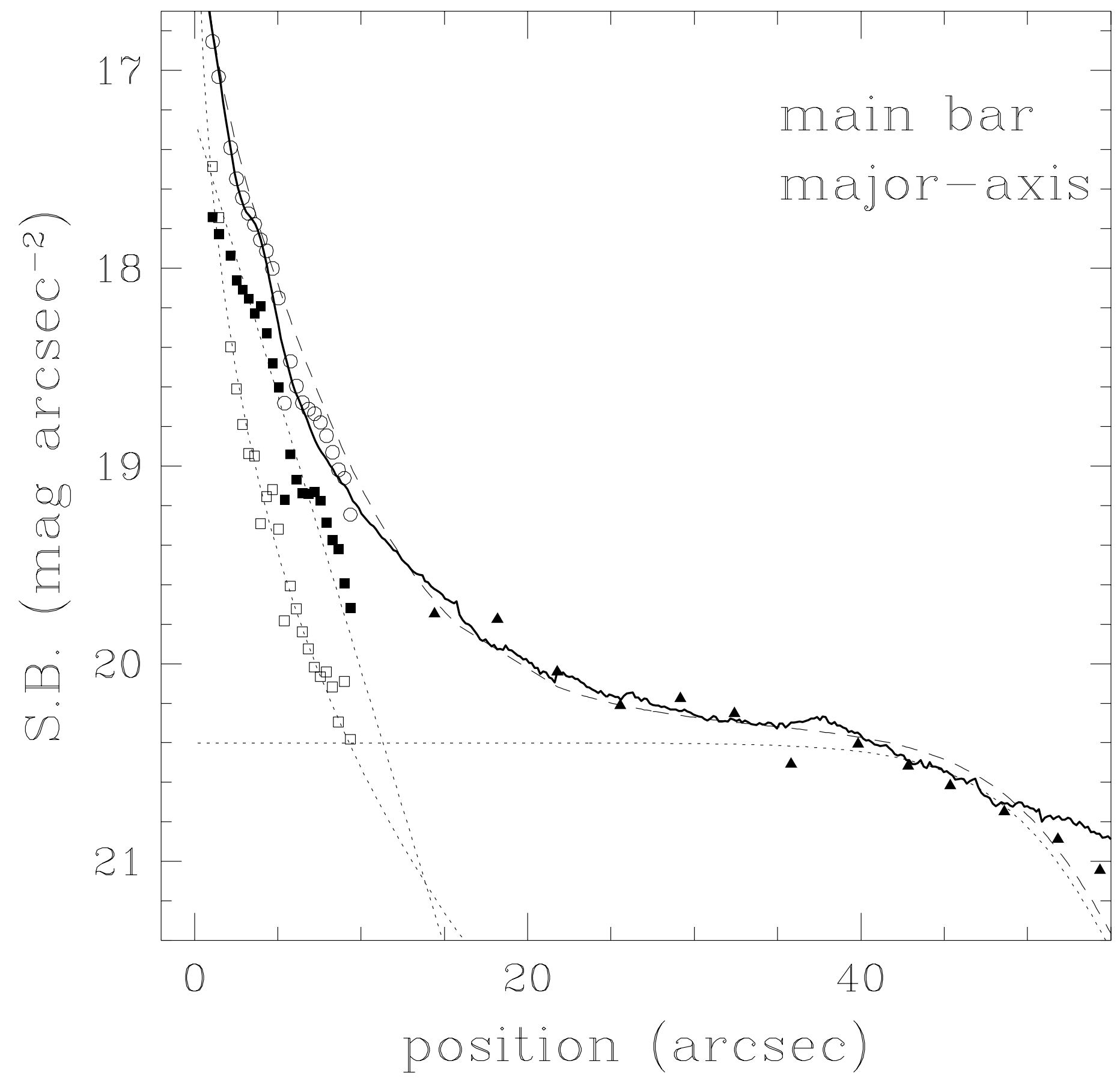

